\title{
ASOCIACIÓN ENTRE CAPACIDAD AERÓBICA Y CALIDAD DE VIDA EN ADULTOS MAYORES DE UNA CIUDAD COLOMBIANA
}

\author{
Marco Chaves-García ${ }^{1, a}$, Carolina Sandoval-Cuellar ${ }^{1, b}$, Pedro Calero-Saa $^{1, a}$
}

\begin{abstract}
RESUMEN
Con el objetivo de determinar la asociación entre la capacidad aeróbica y la calidad de vida relacionada con la salud en adultos mayores, se encuestaron 121 personas en quienes se realizó el protocolo de Bruce modificado, como predictor del consumo máximo de oxígeno $\left(\mathrm{VO}_{2}\right.$ máximo) y el cuestionario de calidad de vida SF-36. El promedio de $\mathrm{VO}_{2}$ máximo para hombres fue de $26,56 \pm 12,67 \mathrm{~mL} / \mathrm{kg} / \mathrm{min}$, y para mujeres de $25,36 \pm 10,72 \mathrm{~mL} / \mathrm{kg} / \mathrm{min}$, obteniéndose asociación significativa entre el sexo y el nivel de $\mathrm{VO}_{2}$ máximo $(\mathrm{p}=0,018)$, las dimensiones de función física y función social fueron las de mayor promedio en el total de la muestra y la capacidad aeróbica explica en un $15,3 \%$ la función física. Se concluye que el nivel pobre de $\mathrm{VO}_{2}$ máximo predomino en ambos sexos y existe asociación entre el nivel de $\mathrm{VO}_{2}$ máximo con las dimensiones de función física, desempeño físico, salud general, vitalidad y función social.
\end{abstract}

Palabras clave: Anciano; Ejercicio, Calidad de vida; Colombia (fuente: DeCS BIREME).

\section{ASSOCIATION BETWEEN AEROBIC CAPACITY AND QUALITY OF LIFE IN OLDER ADULTS IN COLOMBIA}

\begin{abstract}
The objective of this study was to determine the association of aerobic capacity with health-related quality of life in 121 subjects using the modified Bruce protocol as a predictor of maximum oxygen consumption ( $\mathrm{VO}_{2}$ maximum) and the SF- 36 quality of life questionnaire. The average maximum $\mathrm{VO}_{2}$ was $26.56 \pm 12.67 \mathrm{~mL} / \mathrm{kg} / \mathrm{min}$ for men and $25.36 \pm 10.72 \mathrm{~mL} / \mathrm{kg} / \mathrm{min}$ for women. There was a significant association between sex and maximum $\mathrm{VO}_{2}(\mathrm{p}=0.018)$. Physical function and social function were the variables with the highest average values in the total sample, and aerobic capacity explained $15.3 \%$ of the physical function. In conclusion, low maximum $\mathrm{VO}_{2}$ was found in both sexes, and there was a significant association of maximum $\mathrm{VO}_{2}$ with physical function, physical performance, general health, vitality, and social function.
\end{abstract}

Key words: Aged; Exercise; Quality of life; Altitude; Colombia (source: MeSH NLM).

\section{INTRODUCCIÓN}

Actualmente, alrededor del $7 \%$ de la población mundial es adulta mayor. En los países desarrollados este porcentaje es mayor del $15 \%$ y continúa creciendo ${ }^{(1)}$; se proyecta que las personas mayores de 65 años, que para el año 2004 eran 461 millones, pasen a ser 2 mil millones para el año $2050{ }^{(2)}$. La esperanza de vida en Colombia tiene como media nacional los 74 años de edad; por tanto, se espera que para el año 2020 el porcentaje de ciudadanos mayores de 60 años alcance el 12,6\%, valor que aumentará al $23,1 \%$ en el $2050{ }^{(3)}$.

El envejecimiento es un proceso irreversible, natural y progresivo que, además, con el tiempo traerá consigo alteraciones que dificultan la interacción del individuo con su contexto, afectando también habilidades en diferentes áreas biológicas.El proporcionar un envejecimiento activo y saludable permitirá a la población adulta mayor, una mejor calidad de vida ${ }^{(4,5)}$, sumado a esto, la realización de estrategias inherentes al envejecimiento activo, como la práctica de actividad física, permitirá controlar el deterioro de los sistemas osteomuscular, neuromuscular, y cardiovascular, que hacen del ser humano un ser independiente y funcional (4).

En este mismo sentido, la capacidad aeróbica es una cualidad que disminuye con la edad, $10 \%$ por década en personas sedentarias, y $15 \%$ en edades entre 50 y 75 años ${ }^{(6)}$, así como es el mejor indicador para realizar actividad física, la cual trae efectos beneficiosos sobre la presión arterial, los lípidos, la tolerancia a la glucosa, la densidad ósea, la depresión, la calidad de vida ${ }^{(7)}$, entre otras.

\footnotetext{
1 Programa de Fisioterapia, Universidad de Boyacá. Boyacá, Colombia

a Fisioterapeuta; ${ }^{\text {b }}$ Terapeuta física

Recibido: 28/11/2016 Aprobado: 26/07/2017 En línea: 04/12/2017
}

Citar como: Chaves-García M, Sandoval-Cuellar C, Calero-Saa P. Asociación entre capacidad aeróbica y calidad de vida en adultos mayores de una ciudad colombiana. Rev Peru Med Exp Salud Publica. 2017;34(4):672-6. doi: 10.17843/rpmesp.2017.344.2522 
La actividad física puede ser un medio para mantener o mejorar la calidad de vida de las personas mayores, además de ser protector para diversas enfermedades que llevan a un deterioro funcional ${ }^{(8)}$, por lo que es una necesidad en la preservación de la salud en el curso de la vida y estrategia de control de factores ajenos a la enfermedad que afectan la cantidad de salud y la calidad de vida en el envejecimiento ${ }^{(9)}$.

Por lo anterior, se planteó como objetivo el evaluar la asociación entre la capacidad aeróbica y la calidad de vida relacionada con la salud en adultos mayores de los centros vida de la ciudad de Tunja, Colombia.

\section{EL ESTUDIO}

Estudio transversal en una población de 245 adultos mayores que realizan actividad física en los centros vida de la ciudad de Tunja, Colombia, en el año 2015.

Para el cálculo de la muestra se tuvo como referencia la prevalencia del estudio de Correa ${ }^{(10)}$, donde el 18,9\% presentó una clasificación de $\mathrm{VO}_{2}$ excelente. Se utilizó el paquete estadístico EPIDAT versión 3.1, teniendo en cuenta una precisión del $5 \%$ y confiabilidad del $95 \%$ y un tamaño muestral de 121. Para la selección de la muestra se realizó un muestreo aleatorio simple, donde la asignación se hizo por números aleatorios.

Como criterios de inclusión se contemplaron: persona mayor oigual a 65años, estar afiliadoal sistema de seguridad social en salud, afiliado al sistema de identificación de potenciales beneficiarios de programas sociales (SISBEN) y pertenecer a los centros vida del Municipio de Tunja en Colombia. Se excluyeron a los adultos mayores con enfermedades que comprometieran la función cognitiva, lesiones traumáticas agudas, contraindicaciones absolutas permanentes y absolutas temporales al ejercicio físico.

La variable dependiente fue la capacidad aeróbica y se evaluó a través de la aplicación del protocolo de Bruce modificado, como predictor del nivel de consumo máximo de oxígeno en banda sin fin; el desarrollo del protocolo se realizó siguiendo las especificaciones de Heyward (11). Se obtuvieron resultados en $\mathrm{mL} / \mathrm{kg} / \mathrm{min}$, los cuales fueron clasificados en niveles de $\mathrm{VO}_{2}$ según los criterios del Cooper Institure for Aerobics Research. Para mujeres en: pobre entre $\leq 25$ a $26,9 \mathrm{~mL} / \mathrm{kg} / \mathrm{min}$; bueno entre 28 a 29,9 $\mathrm{mL} / \mathrm{kg} / \mathrm{min}$ y excelente $\geq 30$; para hombres: pobre entre $\leq 28$ a $30,9 \mathrm{~mL} / \mathrm{kg} / \mathrm{min}$; bueno entre 32 a $34,9 \mathrm{~mL} / \mathrm{kg} / \mathrm{min}$ y excelente $35 \mathrm{~mL} / \mathrm{kg} / \mathrm{min}{ }^{(11)}$.

Como variables independientes, se evaluó la calidad de vida relacionada con la salud a través del cuestionario de calidad de vida relacionada con la salud (SF-36), el cual reporta datos de fiabilidad y validez según la revisión realizada por

\section{MENSAJES CLAVE}

Motivación para realizar el estudio. Como profesionales encargados de la intervención del Movimiento Corporal humano y ante la realidad mundial de los mayores de 65 años, estamos convocados al mejoramiento del proceso de envejecimiento con alternativas de vida activa.

Principales hallazgos. El presente estudio evidenció asociación entre el nivel de $\mathrm{VO} 2$ máximo con las dimensiones de función física, desempeño físico, salud general, vitalidad y función social,

Implicancias. Los resultados permitirán a profesionales incluir estrategias de intervención hacia la prevención de la salud, minimizando el impacto de los procesos biológicos del envejecimiento, disminuyendo el riesgo de una vejez dependiente y con baja calidad de vida.

Vilagut a 17 estudios que hicieron uso del SF-36, exponiendo UNalfa de Cronbach en las diferentes dimensiones desde 0,94 hasta 0,71 , e indica que para cada dimensión los ítems son codificados, agregados y transformados en una escala que tiene un recorrido desde 0 (el peor estado de salud para esta dimensión) hasta 100 (el mejor estado de salud); puntuaciones superiores o inferiores a 50 indican mejor o peor estado de salud respectivamente ${ }^{(12)}$; adicionalmente, se registraron datos sociodemográficos, así como antecedentes de enfermedades crónicas que afectara los sistemas cardiovascular, pulmonar, musculoesquelético, neuromuscular, tegumentario, metabólico, endocrino u otro.

El análisis de las variables categóricas se realizó a través de la distribución de frecuencias absolutas y relativas, se calcularon medidas de tendencia central y de dispersión para las variables cuantitativas. Se realizó la prueba de chi cuadrado entre las variables sociodemográficas con el nivel obtenido de $\mathrm{VO}_{2}$; se utilizó la prueba no paramétrica rho de Spearman, previa obtención del resultado de distribución según la prueba de Kolmogorov-Smirnov, para encontrar la asociación entre capacidad aeróbica y las dimensiones del cuestionario SF36. Posteriormente, se realizó un modelo de regresión lineal simple entre las dimensiones del cuestionario de calidad de vida SF-36 y la capacidad aeróbica, haciendo comprobación de los supuestos para el desarrollo del modelo. Para el análisis de los datos se utilizó el programa estadístico SPSS 23 ®.

El presente estudio tuvo aprobación del Comité de Bioética de la Universidad de Boyacá.

\section{RESULTADOS}

En general, el promedio de edad fue de 70,9 \pm 5,8 años; 92 participantes fueron mujeres (76\%), el estado civil y nivel educativo más frecuente fueron no tener pareja con un $76,6 \%$ 
Tabla 1. Nivel de capacidad aeróbica según sexo,estado civil yantecedente patológico en adultos mayores de un municipio de Colombia

\begin{tabular}{|c|c|c|c|c|c|}
\hline \multirow[b]{3}{*}{ Variable } & \multicolumn{4}{|c|}{ Nivel de capacidad aeróbica } & \multirow[b]{3}{*}{ Valor de $\mathrm{p}^{*}$} \\
\hline & Total & Pobre & Bueno & Excelente & \\
\hline & n (\%) & n (\%) & n (\%) & n (\%) & \\
\hline \multicolumn{6}{|l|}{ Sexo } \\
\hline Masculino & $29(24,0)$ & $16(55,2)$ & $8(27,6)$ & $5(17,2)$ & 0,01 \\
\hline Femenino & $92(76,0)$ & $53(57,6)$ & $8(8,7)$ & $31(33,7)$ & \\
\hline \multicolumn{6}{|l|}{ Estado civil } \\
\hline Con pareja & $23(19,0)$ & $16(69,6)$ & $1(4,3)$ & $6(26,1)$ & 0,27 \\
\hline Sin pareja & $98(81,0)$ & $53(54,1)$ & $15(15,3)$ & $30(30,6)$ & \\
\hline \multicolumn{6}{|c|}{ Antecedentes patológicos } \\
\hline Sí & $92(76,0)$ & $56(60,8)$ & $11(12,0)$ & $25(27,2)$ & 0,31 \\
\hline No & $29(24,0)$ & $13(44,8)$ & $5(17,3)$ & $11(39,7)$ & \\
\hline
\end{tabular}

* Prueba de independencia (chi cuadrado)

$(n=98)$ y primaria con un $62 \%(n=75)$, respectivamente. El $76 \%$ presentó algún tipo de antecedente personal de enfermedad.

En su mayoría, ambos sexos se clasificaron con un nivel pobre de $\mathrm{VO}_{2}$, seguido del nivel excelente y bueno. Se presentó asociación estadísticamente significativa entre el nivel de capacidad aeróbica con la variable sexo $(p=0,018)$, (Tabla 1).

Con respecto a la calidad de vida relacionada con la salud, para ambos sexos, los dominios de función física y función social fueron los de mayor promedio en relación con las otras dimensiones contempladas en el cuestionario SF36; la media de $\mathrm{VO}_{2}$ máximo por protocolo de Bruce modificado

Tabla 2. Calidad de vida relacionada con la salud y $\mathrm{VO}_{2}$ máximo en adultos mayores de un municipio de Colombia $(n=121)$

\begin{tabular}{lc}
\hline Variable & Media (DE) \\
\hline $\mathrm{VO}_{2}$ máximo $(\mathrm{mL} / \mathrm{kg} / \mathrm{min})$ & $25,7(11,2)$ \\
\hline Dimensión de SF36* & \\
\hline Función física & $70,0(23,5)$ \\
\hline Desempeño físico & $57,9(43,5)$ \\
\hline Dolor corporal & $57,5(28,4)$ \\
\hline Salud general & $53,5(19,6)$ \\
\hline Vitalidad & $61,4(24,5)$ \\
\hline Función social & $72,1(31,3)$ \\
\hline Desempeño emocional & $52,6(43,8)$ \\
\hline Salud mental & $64,5(23,9)$ \\
\hline
\end{tabular}

* Cuestionario SF36 para evaluar calidad de vida.

$\mathrm{DE}$ : desviación estándar; $\mathrm{VO}_{2}$ máximo: consumo máximo de oxígeno o capacidad aeróbica. para el sexo masculino fue de $26,56 \mathrm{~mL} / \mathrm{kg} / \mathrm{min}$ y el femenino de 25,36 mL/kg/min (Tabla 2).

El coeficiente de correlación de Rho de Spearman entre la capacidad aeróbica y las dimensiones de la calidad de vida relacionada con la salud de función física, desempeño físico, salud general, vitalidad y función social mostraron ser estadísticamente significativas (Tabla 3).

La regresión lineal simple presentó una baja influencia de la capacidad aeróbica sobre cada una de las dimensiones de la calidad de vida relacionada con la salud. Según el resultado de $\mathrm{R}$ cuadrado, la máxima influencia presentada fue de un 15,3\% para la dimensión de función física en comparación con las demás.

Tabla 3. Asociación entre capacidad aeróbica y calidad de vida relacionada con la salud en adultos mayores de un municipio de Colombia

\begin{tabular}{lcc}
\hline Dimensión de SF36* & $\begin{array}{c}\text { Capacidad } \\
\text { aeróbica }\end{array}$ & \\
\cline { 2 - 2 } & Rho** $^{*}$ & Valor de p \\
\hline Función física & 0,400 & 0,01 \\
\hline Desempeño físico & 0,229 & 0,01 \\
\hline Dolor corporal & 0,161 & 0,07 \\
\hline Salud general & 0,293 & 0,01 \\
\hline Vitalidad & 0,319 & 0,01 \\
\hline Función social & 0,223 & 0,01 \\
\hline Desempeño emocional & 0,168 & 0,06 \\
\hline Salud mental & 0,141 & 0,11 \\
\hline
\end{tabular}

* Cuestionario SF36 para evaluar calidad de vida relacionada con la salud

** Coeficiente de correlación de Rho de Spearman 


\section{DISCUSIÓN}

El nivel de capacidad aeróbica explica en un 15,3\% la dimensión de función física. Se debe tener en cuenta que existe un declive asociado a estilos de vida inactivos que, a menudo, progresa antes de los 80 años. Aunque la capacidad aeróbica tiende a disminuir a un ritmo entre 5 a $15 \%$ por década, después de los 30 años de edad; los estudios indican que las personas físicamente activas pueden retener una reserva suficiente de aptitud aeróbica para mantener la capacidad funcional durante sus últimos años ${ }^{(13,14)}$. Esta disminución se explica por la falta de práctica de actividad física, que conlleva a una caída en el gasto cardíaco, así como una reducción en la diferencia de oxígeno arteriovenoso, existiendo diferencias entre sexos, ya que son los hombres los que evidencian esta condición más rápidamente ${ }^{(15)}$.

Se evidencian diferencias con los otros estudios que usaron el protocólo de Bruce modificado; en el estudio de Billinger, en adultos mayores de 65 años de la ciudad de Kansas se encontró un $\mathrm{VO}_{2}$ máximo de $22,2 \pm 4,5 \mathrm{~mL} / \mathrm{kg} /$ min (16); así mismo, Nuttamonwarakul reporta en adultos mayores de 60 años con antecedentes de diabetes tipo II de Tailandia, un $\mathrm{VO}_{2}$ máximo de $23,6 \pm 0,5 \mathrm{~mL} / \mathrm{kg} / \mathrm{min}{ }^{(17)}$, de igual manera, Hernández encontró en adultos mayores de Puerto Rico $\mathrm{VO}_{2}$ máximo de $11,6 \pm 3,8 \mathrm{~mL} / \mathrm{kg} / \mathrm{min}{ }^{(18)}$.

Las diferencias encontradas pueden deberse al tipo de actividad física realizada, a la periodicidad de la práctica de actividad física por semana, al tipo de actividad física desarrollada, a las condiciones ambientales y climáticas de las ciudades donde se llevaron a cabo las mediciones, o al antecedente de ser una persona sedentaria o activa, variables que no fueron tenidas en cuenta en el presente estudio, y que podrían estar influenciando, el nivel de capacidad aeróbica en esta población.

Con respecto a la calidad de vida relacionada con la salud contrastando con la población de Medellín Colombia, Estados Unidos y España, el ítem de vitalidad supera a lo encontrado en la población estadounidense; de igual manera, supera en los ítems de función física, desempeño físico, dolor corporal, vitalidad, función social y salud mental, a la población de Medellín, pero en ninguno supera a la población española ${ }^{(19)}$. Patiño encontró que el dominio de función física fue el único que se asoció con el resultado de capacidad aeróbica en adultos de la ciudad de Medellín (20), siendo similar a los resultados de la presente investigación. Estos resultados pueden estar influenciados por la presencia de uno a más antecedentes de enfermedad, a la constitución de una familia, a tener una vejez solitaria o acompañada, a la facilidad de acceder a servicios de salud y de actividades de promoción y prevención, a tener una envejecimiento activo y saludable, aunque es importante tener en cuenta que en los hombres del presente estudio, la mayor puntuación se presentó en el dominio de salud física, y en las mujeres al dominio de salud mental, lo que evidencia diferentes intereses para ambos sexos.

Como limitaciones del estudio, está la presencia de enfermedades crónicas no transmisibles en los adultos mayores, la cual puede explicar la calidad de vida reportada; también se considera la baja influencia de la capacidad aeróbica sobre las dimensiones de la calidad de vida relacionada con la salud, debido al tamaño de muestra del estudio y el posible sesgo de memoria por parte de los participantes.

Adicionalmente, para el estudio se creyó conveniente no considerar a las variables edad, estado civil o antecedentes patológicos como intervinientes, atendiendo a que estas podrían afectar la asociación entre calidad de vida relacionada con la salud y la capacidad aeróbica. Otra limitación es que incluida la muestra ya formaban parte de un programa de adultos mayores, lo que podría establecer basalmente una mejor capacidad aeróbica de otros adultos mayores, así como una mejora en la calidad de vida por ser un grupo intervenido desde una red de apoyo. Se recomiendan más estudios en los que se contemplen las aptitudes físicas que influyen para que un adulto mayor goce de un envejecimiento activo y saludable, así como estudios con un diseño metodológico que permita el seguimiento.

En conclusión la capacidad aeróbica y la calidad de vida relacionada con la salud son condiciones que están asociadas a un declive que progresa con la edad y a estilos de vida inactivos que dependerán de valores, creencias, contexto cultural, historia personal, subjetividad, aspectos físicos, emocionales y sociales en cada una de las etapas de la vida.

Contribuciones de autoría: MCG participó en la recolección de resultados y redacción de artículo. CSC realizó el análisis e interpretación de datos. PCS participó en la recolección de resultados y revisión crítica de artículo.

Fuentes de financiamiento: estudio financiado por la Universidad de Boyacá.

Conflictos de interés: los autores declaran no tener conflictos de interés

\section{REFERENCIAS BIBLIOGRÁFICAS}

1. Landinez P, Parra N, Contrera K, Castro Ángel. Proceso de envejecimiento, ejercicio y fisioterapia. Rev Cub Salud Pública. 2012;38(4):562-80.
2. Clegg A, Young J, Iliffe S, Rikkert MO, Rockwood K. Frailty in elderly people. The lancet. 2013;281:752-62. doi: 10.1016/ S0140-6736(12)62167-9
3. Arrubla-Sanchez D. Política social para el envejecimiento: el ( $\sin )$ sentido de los argumentos. Rev. Gerenc. Polit. Salud. 2010;9(19):229-42. 
4. Pérez V, Sierra F. Biología del envejecimiento. Rev. méd. Chile. 2009;138(2):296-302.

5. Sangeeth GS, Solomon MD. Quality of life among elderly in non-institutional care. Int J Sci Res. 2015;4(2):1462-67.

6. Hollenberg M, Yang J, Haight TJ, Tager IB. Longitudinal changes in aerobic capacity: implications for concepts of aging. J Gerontol A Biol Sci Med Sci. 2006;61(8):851-858.

7. Fleg JL. Aerobic exercise in the elderly: a key to successful aging. Discov Med. 2012;13(70):223-8.

8. Choi M, Prieto-Merino D, Dale C, Nuesch E, Amazu A, Bowling A, et al. Effect of changes in moderate or vigorous physical activity on changes in healthrelated quality of life of elderly British women over seven years. Qual Life Res. 2013;22(8):2011-20. doi: 10.1007/ s11136-012-0332-2

9. García C. Evaluación y cuidado del adulto mayor frágil. Rev. Med. Clin. Condes. 2012;23(1):36-41

10. Correa J, Gámez E, Ibáñez M, Rodríguez K. Aptitud física en mujeres adultas mayores vinculadas a un programa de envejecimiento activo. Salud UIS. 2011;43(3):236-269
11. Heyward V, Gidson A. Advanced Fitness Assessment and Exercise Prescription. Susan $\mathrm{H}$, editors. Preliminary health screening and risk classification. New Mexico: Human Kinetics; 2014. p. 92-94.

12. Vilagut G, Ferrer, M, Rajmil L. Rebollo P, Permanyer-Miranda, G. Quintana J, et al. El cuestionario de salud SF-36 español: una década de experiencia y nuevos desarrollos. Gac Sanit. 2005;19(2):135-50.

13. Rikli R, Jones J. Senior Fitness Test Manual. Tocco A, editor. The senior fitness test. United States: Human Kinetics; 2013.p. 15.

14. Hayashi D, Gonçalves CG, Parreira RB, Fernández KB. Teixeira DC, Silva $\mathrm{RA}$, et al. Postural balance and physical activity in daily life (PADL) in physically independent older adults with different levels of aerobic exercise capacity. Arch Gerontol Geriatr. 2012;55(2):480-5. doi: 10.1016/j.archger.2012.04.009

15. Milanovic Z, Pantelic S, Trajkovic N, Sporis G, Kostic R, James N. Age-related decrease in physical activity and functional fitness among elderly men and women. Clin Interv Aging. 2013;8:549-56.

16. Billinger S, Vidoni E, Morris J, Thyfault J, Burns J. Exercise Test Performance Reveals Support of the Cardiorespiratory Fitness
Hypothesis. J Aging Phys Act. 2017; 25(2):240-46. doi:10.1123/japa.20150321.

17. Nuttamonwarakul A, Amatyakul S, Suksom D. Twelve weeks of aqua-aerobic exercise improve health related physical fitness and glydemic control in elderly patiens with type 2 diabetes. JEPonline. 2012;15(2):64-70.

18. Hernández $\mathrm{O}$, Ramirez F. Programa de ejercicio estructurado es viable y mejora la capacidad funcional en adultos mayores en Puerto Rico. Pensar en Movimiento: Revista de ciencias del ejercicio y la salud. 2014;12(2):1-15.

19. García H, Vera Claudia, Lugo L. Calidad de vida relacionada con la salud en Medellín y su área metropolitana, con aplicación del SF-36. Rev. Fac. Nac. Salud Pública. 2014;32(1):26-39.

20. Patiño F, Arango E, Lopera N, Ortiz N, Perez E, Santamaría J, et al. Calidad de vida relacionada con la salud en usuarios de un programa de actividad física. Iatreia. 2011;23(3):238-49

Correspondencia: Marco Antonio Chaves García Dirección: Transversal 2a \# 64-74 Tunja, Boyacá, Colombia.

Correoelectrónico:machaves@uniboyaca.edu.co

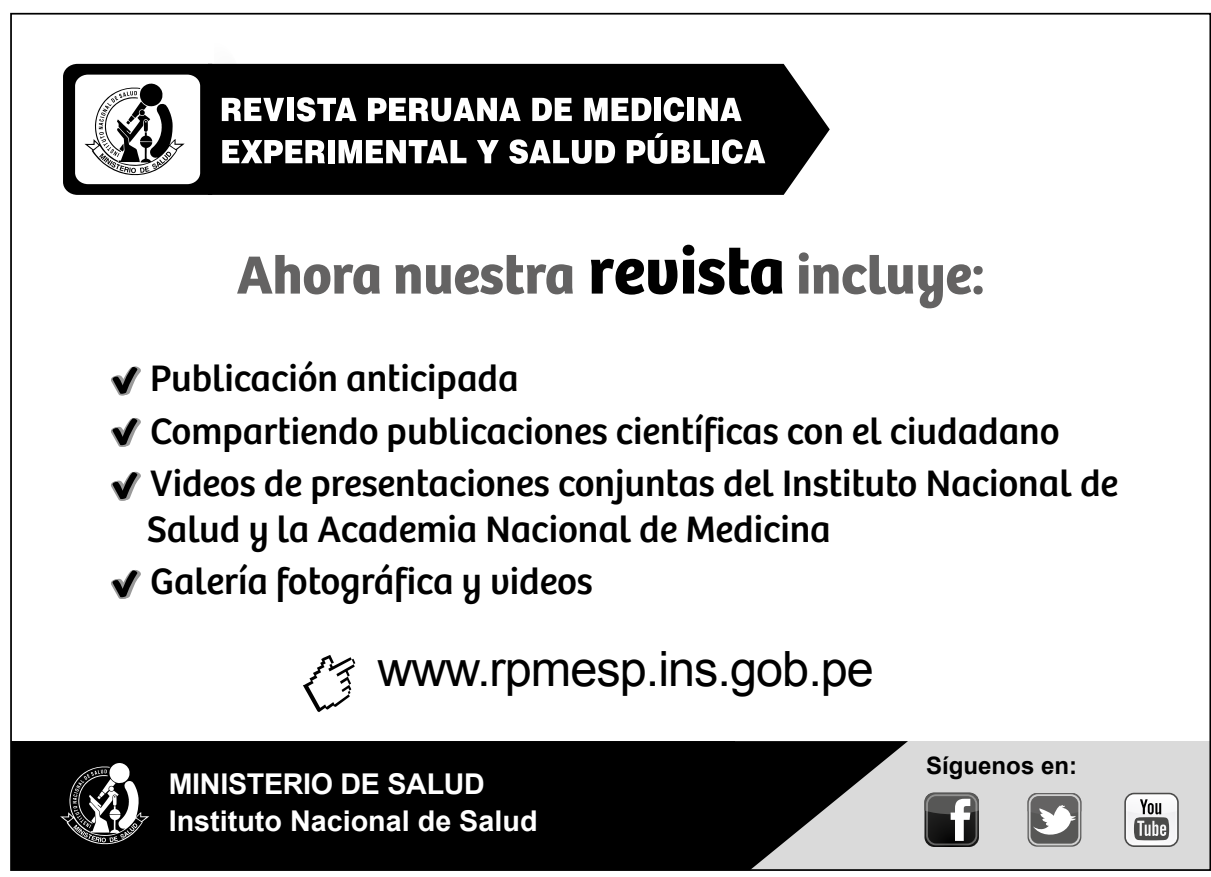

